\title{
Presencia de Triatoma sordida Stäl, 1859 én ecotopos urbanos de la ciudad de Corrientes, Argentina*
}

\section{Presence of Triatoma sordida Stäl, 1859 in Corrientes city urban ecotopes}

\author{
María E. Bar**, Elena B. Oscherov**, Miryam P. Damborsky**
}

\begin{abstract}
BAR, M.E. et al. Presencia de Triatoma sordida Stäl, 1859 en ecotopos urbanos de la ciudad de Corrientes, Argentina. Rev. Saúde Pública, 27: 117-22, 1993. Con el objeto de verificar la presencia de $T$. sordida Stäl 1859, en ecótopos urbanos de la ciudad de Corrientes, Argentina se tomaron como base un hábitat extradomiciliario y la vivienda humana. En el palomar situado en el Parque Mitre fue realizado un censo completo de población de $T$. sordida para lo cual fueron disecados 400 nidos de paloma (Columbia livia). Los ejemplares de triatominos detectados en las viviendas fueron enviados por los pobladores al Laboratorio de Artrópodos. Los triatominos fueron determinados sistemáticamente y por clase de edad. La materia fecal de los mismos fue examinada al microscopio para la identificación del Trypanosoma cruzi. En el palomar se colectaron: 6 huevos, 45 ninfas de primer estadío, 110 ninfas de segundo estadío, 226 ninfas de tercer estadío, 303 ninfas de cuarto estadío, 1.103 ninfas de quinto estadío, 12 hembras y 11 machos. Se constató la presencia de esta especie en 27 viviendas localizadas en 8 barrios de la Ciudad. Se capturaron 13 huevos, 33 ninfas de diferentes estadíos, 27 hembras y 9 machos, totalizando 82 individuos. Ningún triatomino resultó infectado por $T$. cruzi. Los adultos de $T$. sordida se desplazaron hacia el domicilio en primavera y verano. Es de destacar que el $33 \%$ del material hallado en las viviendas correspondió a hembras. Se ha comprobado la adaptación de $T$. sordida a un hábitat extradomiciliario y su tendencia a invadir la vivienda humana, hecho que se debería ser tenido en cuenta en los programas de control de vectores.
\end{abstract}

Descriptores: Triatoma. Ecologia de vectores.Domiciliación.

\section{Introduccion}

El potencial de infestación del ambiente humano por triatominos fue estudiado teniendo presente el rol que desempenan los focos extradomiciliarios en la dispersión $n^{4}$. Triatoma sordida es una especie que se encuentra asociada a nidos de aves ${ }^{3}$ y está bien representada en biótopos naturales y en hábitats peridomiciliarios, desde los cuales puede invadir el domicilio, particularmente cuando $T$. infestans ha sido eliminada ${ }^{11}$. Al respecto, se ha constatado que los adultos de esta especie tienen vuelos de dispersión ${ }^{12}$ y colonizan hábitats artificiales desde sus biótopos naturales, situados a distancias de $250-600 \mathrm{~m}^{6}$.

En el mesenterón de T. sordida, Panstrongylus megistus y Rhodnius neglectus predomina sangre

\footnotetext{
* Investigación subsidiada por la Secretaría de Ciencias y Técnica de la Nación. Resolución N². D-0120-1597/84.

** Cátedra de Artrópodos. Facultad de Ciencias Exactas y Naturales y Agrimensura. Universidad Nacional del Nordeste - Corrientes - Argentina.
}

Separatas/Reprints: M.E. Bar - Calle 9 de Julio, 1449 - 3400 Corrientes - Argentina de ave, pero frecuentemente estas especies incluyen en sus comidas sangre de otros hospedadores $^{7,8}$. La detección de sangre humana y de animales silvestres sugieren que se producen movimientos migratorios entre ecótopos naturales $\mathrm{y}$ artificiales ${ }^{1,2}$.

La diseminación de los triatominos hacia la vivienda humana estaría favorecida por la atracción que eje:cen sobre éstas las fuentes de luz artificial $^{14}$ y por la presencia en los edificios o en sus cercanías, de refugios favorables, tales como los nidos de $P$ asser domesticus, infestados por $T$. sordida, en los techos de las viviendas ${ }^{4}$.

El objetivo del presente trabajo fue estimar la magnitud de infestación por $T$. sordida de un ecótopo extradomiciliario urbano, localizado en la ciudad de Corrientes, Argentina y su significado como fuente de infestación del hábitat doméstico.

\section{Material y Metodo}

Para verificar la presencia de $T$. sordida en el hábitat extradomiciliario fue investigado un palomar ubicado en el parque Mitre de la ciudad de 
Corrientes, Argentina; mientras que la infestación dei ámbito doméstico fue analizada en base al envio de ejemplares por parte de los pobladores.

En el palomar fue realizado un censo completo de población em mayo de 1984, requiriendo 12 días de trabajo, con un esfuerzo de 6 personas durante $6 \mathrm{~h}$ diarias. Para la búsqueda de triatominos se emplé el método captura/hora/hombre ${ }^{15}$.

El palomar no había sido fumigado con anterioridad al censo, según autoridades del Servicio Nacional de Chagas y de la Municipalidad de la ciudad de Corrientes.

El palomar está situado aproximadamente a $140 \mathrm{~m}$ de la margen izquierda del Río Paraná; es de forma octogonal, cada una de sus caras mide $3,70 \mathrm{~m}$ de ancho por $5 \mathrm{~m}$ de altura, con cúspide de $7 \mathrm{~m}$. Sus paredes son de mampostería y el techo de madera y tejas; cada pared tiene una capacidad para albergar 75 nidos (Fig. 1 y 2).

Los nidos constan de una base de espesor variable de materia fecal, tierra y desechos, consti-

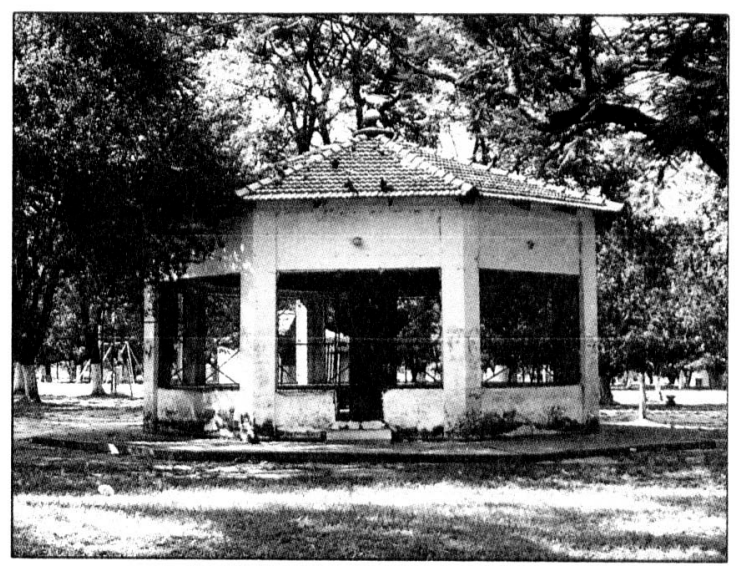

Figura 1. Vista general del palomar.

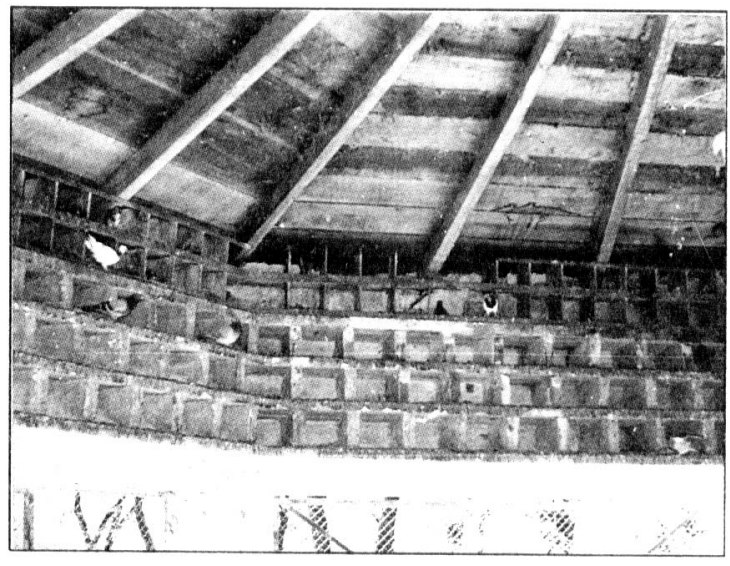

Figura 2. Detalle de los compartimientos.

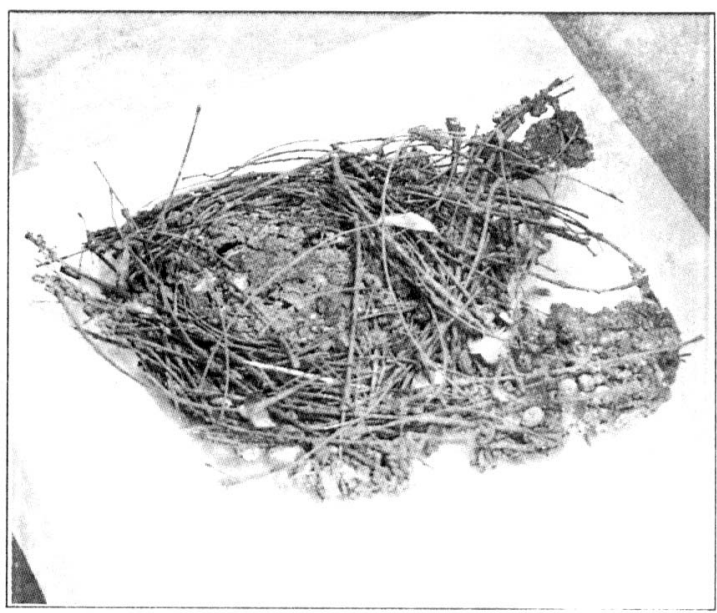

Figura 3. Nido de Columbia livia.

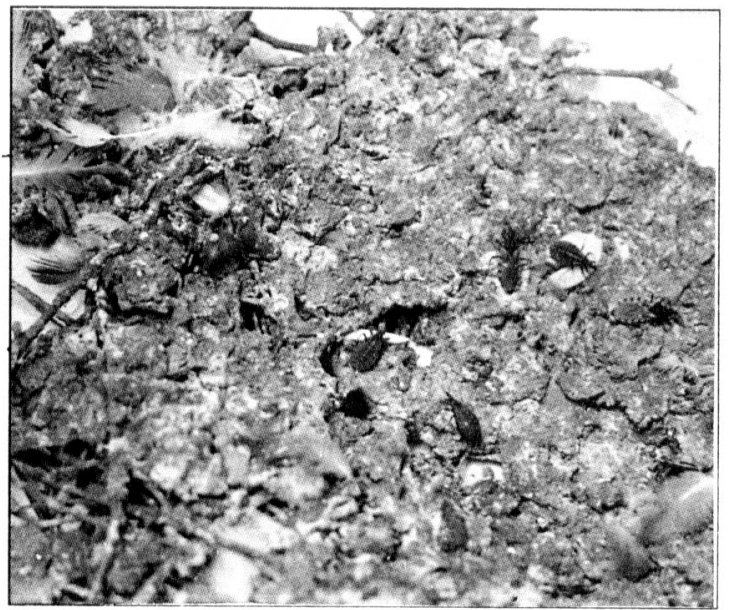

Flgura 4. Base de un nido de paloma.

tuyendo una trama compacta sobre la cual se ubica la cámara de cría. (Fig. 3 y 4).

Los 400 nidos presentes fueron disecados en forma gradual y sistemática, sin utilizarse irritantes químicos para movilizar a los triatominos.

El total de ejemplares de $T$. sordida, enviados espontáneamente por los pobladores al Laborato. rio de Artrópodos*, desde 1983 a 1987, fue considerado una muestra. Dicho material provenía de diferentes domicilios localizados en áreas urbanas consolidadas de la ciudad de Corrientes. Con posterioridad al hallazgo, cada vivienda fue cuidadosamente analizada. Estas no eran precarias; sus componentes estructurales eran similares entre si, con buena instalación de servicios, paredes de mampostería y techos de chapas de metal.

De la Facultad de Ciencias Exactas y Naturales y Agrimensura. Universidad Nacional del Nordeste. 
Los insectos fueron determinados sistemáticamente por estado de desarrollo y sexo $0^{3,9}$. Para la identificación del Trypanosoma cruzi, las heces de los triatominos obtenidas por compresión abdominal, y en caso necesario por disección, fueron diluidas en solución salina de $\mathrm{CINa}$ al $0,85 \%$, observándose al microscopio con 400X. El estado nutricional de los ejemplares fue determinado mediante la observación externa de la silueta del promesenterón y su grado de vaciamiento ${ }^{10}$.

\section{Resultados}

En el palomar fueron colectados ejemplares vivos y muertos. La población viva ascendió a 1.856 ejemplares. La composición etaria y el número de individuos de cada clase de edad se presentan en la Figura 5.

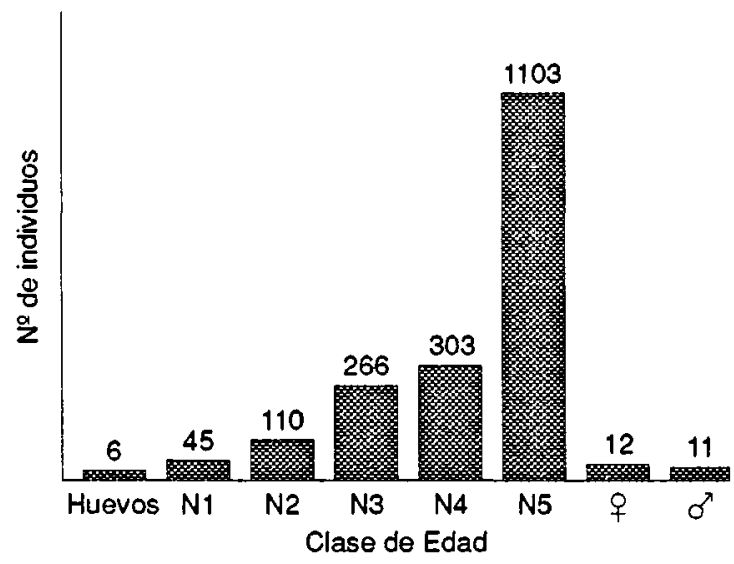

Figura 5. Población de T. sordida colectada en el palomar, discriminada por estados de desarrollo.

Se colectaron 64 ejemplares muertos, identificados como una ninfa de cuarto estadio, 13 ninfas de quinto, 24 hembras y 20 machos. No fue posible la determinación sexual de seis adultos, dado el grado de deterioro.

Respecto a la localización de los individuos en los nidos, las ninfas de tercero, cuarto y quinto estadío y los adultos se hallaban refugiados en la superficie externa de los mismos y en las paredes de los compartimientos, mientras que las ninfas de primero y segundo estadíos permanecian en las partes más internas del sustrato.

Los ejemplares de $T$. sordida recibidos provenían de 27 domicilios. Estos están situados en los siguientes barrios: Cambá Cuá, La Rosada, Centro, Libertad, San Martín, Berón de Astrada, San Benito y Deportes (Figura 6). Las viviendas positivas están situadas a distancias que oscilan entre 300 y 2.760 metros del palomar.

Al Laboratorio de Antrópodos fueron remitidos 82 ejemplares vivos, principalmente en primavera y verano (octubre a marzo), durante cuatro años. Cabe resaltar que el 33\% del material correpondía a hembras, las que estaban fecundadas ya que ovipusieron huevos fértiles. Las mismas fueron clasificadas como adultos ayunados tipo 1 (Montenegro $^{10}, 1983$ ).

El número de ejemplares detectados, discriminados por vivienda y estado de desarrollo se especifica en la Tabla 1. En el $67,7 \%$ de las viviendas fue hallado un sólo ejemplar, en los domicilios restantes el número de individuos varió de 2 a 28. Por otra parte, en 26 viviendas se colectaran hembras, hallándose machos únicamente en siete de ellas.

Tabla 1. Frecuencia del número de ejemplares de $T$. sordida detectados en las viviendas y clase de edad de los mismos.

\begin{tabular}{|c|c|c|c|c|c|c|c|}
\hline \multirow{2}{*}{$\begin{array}{l}\text { № de ejem- } \\
\text { plares por } \\
\text { vivienda }\end{array}$} & \multirow{2}{*}{ Frec. } & \multirow[b]{2}{*}{$(\%)$} & \multicolumn{5}{|c|}{ Ejemplares por edad de desarrollo } \\
\hline & & & Huevos & Ninfas & $\begin{array}{l}\text { Hem- } \\
\text { bras }\end{array}$ & $\begin{array}{l}\text { Ma- } \\
\text { chos }\end{array}$ & Total \\
\hline 1 & 18 & 66,7 & - & - & 14 & 4 & 18 \\
\hline 2 & 5 & 18,5 & - & - & 9 & 1 & 10 \\
\hline 3 & 1 & 3,7 & - & - & - & 3 & 3 \\
\hline 9 & 1 & 3,7 & - & 6 & 2 & 1 & 9 \\
\hline 14 & 1 & 3,7 & 13 & - & 1 & - & 14 \\
\hline 28 & 1 & 3,7 & - & 27 & 1 & - & 28 \\
\hline Total & 27 & 100,0 & 13 & 33 & 27 & 9 & 82 \\
\hline
\end{tabular}

El $45 \%$ de la muestra correspondió a dos depósitos anexos a la vivienda, mientras que el resto de los triatominos fue capturado en galerías $(22 \%)$, lavaderos $(17 \%)$ y dormitorios $(16 \%)$.

Los triatominos provenientes tanto del palomar como de las viviendas no estaban infectados por Trypanosoma cruzi.

\section{Conclusion y Discusion}

Tanto hábitats domiciliarios como extradomiciliarios de la ciudad de Corrientes resultaron positivos a $T$. sordida.

Respecto a la población presente en el momento de censar el palomar, llama la atención el elevado número de ninfas de quinto estadío en relación a las otras edades, particularmente adultos, porque ello significa una alta mortalidad o una alta tasa de dispersión, lo que entraña la probabilidad de infestación de ambientes vecinos. Respecto a lo ante dicho, la movilidad de $T$. sordida fue confirmada al 


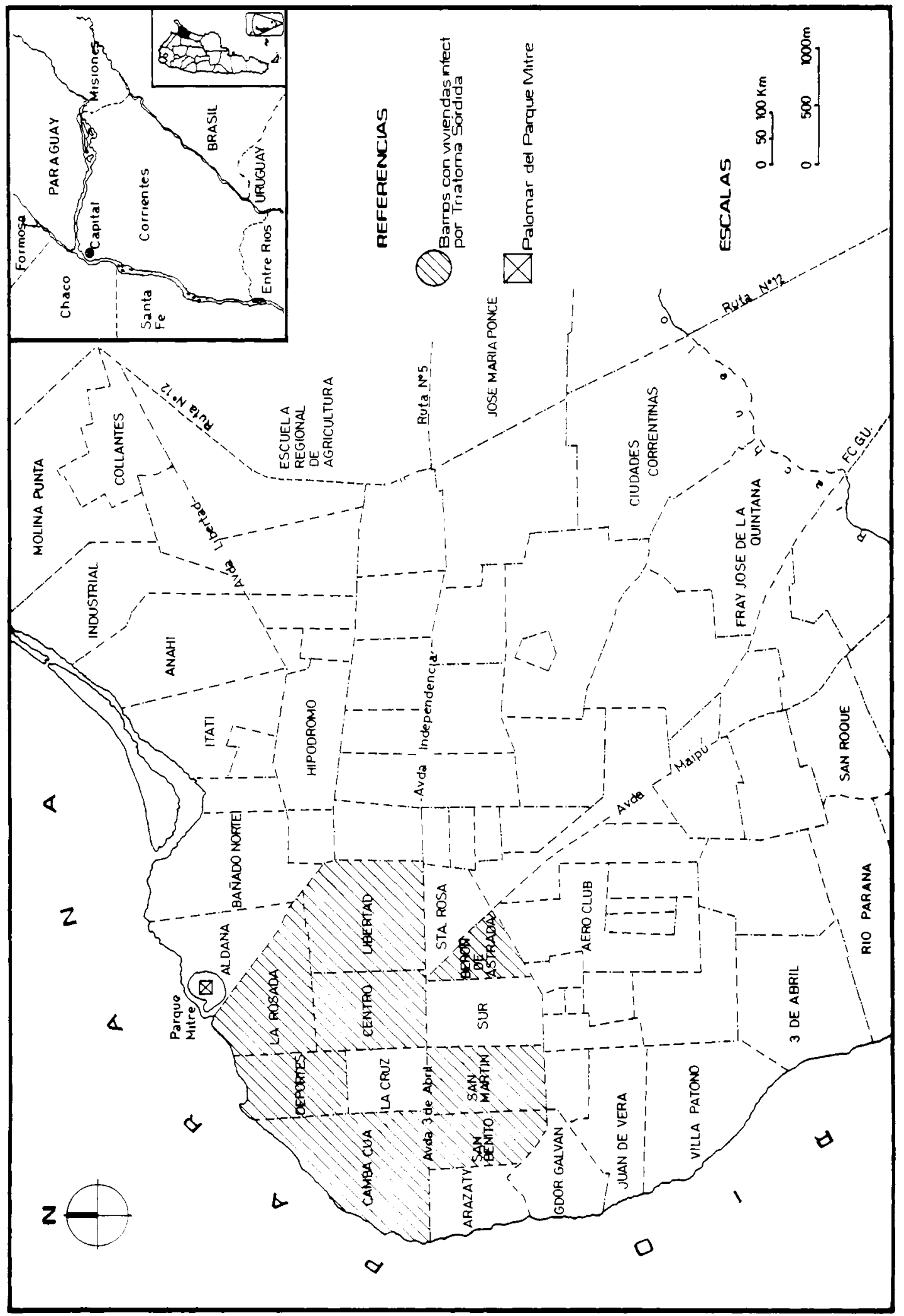

Figura 6. Plano de la ciudad de Corrientes, provincia de Corrientes, Argentina. Localización de los barrios infestados por T. sordida. 
abandonar los adultos los ecótopos artificiales que habían colonizado espontáneamente (Forattini et al. $\left.{ }^{5}, 1977\right)$.

Todos los ejemplares de $T$. sordida fueron hallados en las viviendas en primavera y verano. Coincidentemente Forattini et al. ${ }^{5}$, (1977) comprueban la existencia de un ritmo anual de dispersión de adultos (enero-marzo) desde sus ecótopos de origen, sustentado en el hecho de que el vuelo de $T$. sordida, con una capacidad máxima de $700 \mathrm{~m}$, es una actividad predominante en los meses de primavera y verano (Zeledon $\left.{ }^{16}, 1983\right)$.

Algunos domicilios infestados por $T$. sordida están situados a más de $2.700 \mathrm{~m}$ del palomar, distancia superior al rango de vuelo de esta especie, lo que indicaría la presencia de otros biótopos no conocidos a partir de los cuales se dispersaría.

$T$. sordida invade las moradas llevada por la necesidad de supervivencia, al encontrar en las mismas un nuevo ecótopo que ofrece al hombre como fuente de alimento alternativa ${ }^{4}$. Los ejemplares hallados en los domicilios estaban ayunados. Al estudiar en San Juan (Argentina), el vuelo de dispersión de $T$. infestans Schweigman et al. ${ }^{13}$ (1988) observan que ninguno de los ejemplares atrapados estaba alimentado.

Los moradores de las viviendas con presencia de vinchucas, manifestaron que los ejemplares llegaban a sus domicilios de noche y atraidos por la luz artificial, en concordancia con lo verificado para $T$. infestans ${ }^{13}$. Schofield et al. ${ }^{12}$ (1991) comprueban que la actividad de vuelo de $T$. sordida es principalmente nocturna, pudiendo efectuar además de los vuelos de dispersión, vuelos triviales de hasta $5 \mathrm{~m}$ y una altura máxima de $1 \mathrm{~m}$.

Rocha e Silva et al. ${ }^{11}$ (1977) sostienen que la proximidad de $T$. sordida al domicilio se ve favorecida por la presencia de pájaros en el mismo. También fue comprobado que los gorriones transportan a ninfas de $T$. sordida de una vivienda a otra $^{4}$. Por lo antes expuesto, se plantea el riesgo de infestación que representan los nidos de $C$. livia en los edificios de la ciudad de Corrientes.

En la muestra domiciliaria llama la atención el mayor porcentaje de hembras.

En cuanto a la magnitud de la infestación por $T$. sordida se puede decir que fue importante en el palomar y relativa en la vivienda humana, a excepción de dos domicilios que estarían colonizados, dada la composición de las poblaciones detectadas.

Se ha constatado una vez más la adaptación de $T$. sordida a diferentes ecótopos y su tendencia a invadir la vivienda, hecho que debería ser tenido en cuenta en los programas de control de vectores.

\section{Agradecimientos}

A la Prof. Viviana Repáraz y al Prof. José L. Romero por su contribución en la disección de nidos de palomas, al Lic. Anibal R. Bar por su participación en la colección y análisis de heces de los triatominos.

BAR, M. E. et al. Presença de Triatoma sordida Stäl 1859, em ecótopos da cidade de Corrientes da República Argentina. Rev. Saúde Pública, 27: 117-22, 1993. Com o objetivo de verificar a presença de $T$. sordida em ecótopos urbanos da cidade de Corrientes, Argentina, tomaram-se como base um habitat extradomiciliário e a domicnilio. Em um pombal situado no parque Mitre foi realizado censo da população de $T$. sordida para o qual foram dissecados 400 ninhos de pombas (Columbia livia). Os exemplares detectados no domicílio foram enviados por moradores ao Laboratório de Artrópodos. Os triatomíneos foram determinados sistematicamente e por classe de idade. A matéria fecal dos mesmos foi examinada ao microscópio com 400 aumentos para a identificação do Trypanosoma cruzi. No pombal coletaram-se 1.920 exemplares de $T$. sordida, população constituída por ovos, ninfas e adultos. Em 27 domicílios foi constatada a presença desta espécie, capturando-se 13 ovos, 33 ninfas de diferentes estádios, 27 fêmeas e 9 machos. Nenhum triatomíneo resultou infectado por $T$. cruzi. Os adultos de $T$. sordida deslocaramse ao domicílio na primavera e verão, sendo que $33 \%$ do material encontrado nos domicílios correspondeu a fêmeas. Comprovaram-se a adaptação de $T$. sordida a um habitat extradomiciliário e a sua tendência a invadir domicinio, fatos que se deveriam ter em conta nos programas de controle de vectores.

Descritores: Triatoma. Ecologia de vetores. Domiciliação.

BAR, M. E. et al [Presence of Triatoma sordida Stäl, 1859 in Corrientes city urban ecotopes]. Rev. Saúde Pública, 27: 117-22, 1993. With the intention of verifying the presence of $T$. sordida Stál 1859 in urban ecotopes in Corrientes city, Argentina, both, human dwellings and an extradomiciliary habitat were taken as a basis. A complete $T$. sordida population census was carried on in Mitre Park pigeon house and 400 pigeon nests (Columbia livia) were dissected. The triatomineos samples that had been detected in human dwellings were sent to the Arthropods Laboratory by their inhabitants. These triatomineos were classified systematically and according to their age class. Their feces were examined under a microscope $400 \mathrm{x}$, so as to identify Trypanosoma cruzi. Six eggs, 45 first-instar nymphs, $110 \mathrm{sec}$ ond-instar nymphs, 226 third-instar nymphs, 303 fourthinstar nymphs, 1.103 fifth-instar nymphs, 12 females and 11 males were collected from the pigeon house. The presence of this species was verified in the 27 houses, situated in 8 different neighborhoods in the city; 13 eggs, 33 different instar nymphs, 27 females and 9 
males were captured, 82 samples altogether. No triatomineo was infected by $T$. cruzi. $T$. sordida adults moved towards the houses in Spring and Summer. Of the material found in human dwellings $33 \%$ was female. The adaptation of $T$. sordida to extra-domestic habitats and their tenfency to invade human houses has thus been verified. This fact should be taken into account in vector control programs.

Keywords: Triatoma. Ecology, vectors. Domiciliation.

\section{Referênclas Blbliográficas}

1. BARRETO, M.P. Estudo sobre reservatórios e vectores silvestres do Trypanosoma cruzi. XXXI. Observaçöes sobre a associação entre reservatórios e vectores, com especial referência a Região Nordeste do Estado de São Paulo. Rev. Bras. Biol., 28: 481-94, 1968.

2. BARRETO, M.P. Estudos sobre reservatórios e vectores silvestres do Trypanosoma cruzi. XLV. Inquérito preliminar sobre triatomineos silvestres no sul do Mato Grosso, Brasil. Rev. Bras. Biol., 31: 225-33, 1971.

3. BREWER, M.; GARAY, M.; GORLA, D.; MURUA, F. \& FAVOT, R. Caracterización de los estadíos ninfales del género Triatoma Laporte 1833. II. Triatoma platensis Neiva 1913, Triatoma delpontei Romaña y Abalos 1947, Triatoma sordida Stal 1859, (Hemiptera, Reduviidae). Rev. Soc. Ent. Argentina, 42(1/4): 219-41, 1983.

4. FORATTINI, O.P.; ROCHA E SILVA, E.O.; FERREIRA, O.A.; RABELLO, E.X. \& PATTOLI, D.G.B. Aspectos ecológicos da tripanossomiase americana. III. Dispersão local de triatomineos, com especial referência ao Triatoma sordida. Rev. Saúde Pública, 5: 193-05, 1971.

5. FORATTINI, O.P.; SANTOS, J.L.F.; FERREIRA, O.A.; ROCHA E SILVA, E.O. \& RABELLO, E.X. Aspectos ecológicos da tripanossomíase americana. X. Dados populacionais das colônias do Panstrongylus megistus e do Triatoma sordida espontaneamente desenvolvidas em ecotopos artificiais. Rev. Saúde Pública, 11:362-74, 1977.

6. FORATTINI, O.P.; FERREIRA, O.A.; ROCHA E SILVA, E.O. \& RABELLO, E.X. Aspectos ecologicos da tripanossomíase americana. XV. Desenvolvimento, variação e permanência de Triatoma sordida, Panstrongylus megistus e Rhodnius neglectus em ecotopos artificiais. Rev. Saúde Pública, 13: 220-34, 1979.
7. FORATTINI, O.P.; BARATA, J.M.S.; SANTOS, J.L.F. \& SILVEIRA, A.C. Hábitos alimentares, infecção natural e distribuição de triatomíneos domiciliados na região central do Brasil. Rev. Saude Pública. 16: 171-204, 1982.

8. FREITAS, J.P.L. de; SIQUEIRA, A.F.; FERREIRA, O.A. Investigações epidemiologicas sobre triatomineos de hábitos domésticos e silvestres com auxilio da reação de precipitinas. Rev. Inst. Méd. Trop. S. Paulo, 2: $90-$ 9, 1960.

9. LENT, H. \& WYGODZINSKY, P. Revision of Triatominae (Hemiptera. Reduviidae) and their significance as vectors of Chagas'disease. Bull. Am. Mus. Nat., 163 (3): 520, 1979.

10. MONTENEGRO, S.S.C. de Determinación de reservas alimenticias en Triatoma infestans Klug, 1834 (Hemiptera, Reduviidae) en base a caracteres extemos. I. Adultos. Physis, 41 (101): 159-67, 1983.

11. ROCHA e SILVA, E.O. da; SOUZA, J.M.P. de; ANDRADE, J.C.R. de; MELLO, C. da S.; FERREIRA, $O$.A. Preferéncia alimentar (entre sangue humano e ave) dos Triatoma sordida encontrados em casas habitadas da região norte do Estado de São Paulo. Brasil. Rev. Saúde Pública 11: 258-69, 1977.

12. SCHOFIELD, C.J.; LEHANE, M.J.; Mc. EWAN, P.; CATALA, S.S.; GORLA, D.E. Dispersive flight by Triatoma sordida. Trans. R. Soc. Trop. Med. Hyg., 85: 676-8, 1991.

13. SCHWEIGMANN, N.; VALLVE, S.; MUSCIO, O.; GHILLINI, M.; ALBERTI, A.; WISNIVESKY-COLLI, C. Dispersal flight by Triatoma infestans in an arid area of Argentina. Med. Vet. Entom., 2: 1988. [En prensa].

14. USINGER, R.L. The Triatominae of North and Central America and the West Indies and their public health significance. Public Health Bull., (288): 81, 1944.

15. WISNIVESKY-COLLY, C.; PAULONE, I.; PEREZ, A.; CHUTT, R.; GUALTIERI, J.; SOLARZ, N.; SMITH, A.; SEGURA, E. A new tool for continuous detectio of the presence of Triatominae bugs, vectors of Chagas'disease in rural house-holds. Medicina, Buenos Aires, 47: 45-50, 1987.

16. ZELEDON, $R$. Vectores de la enfermedad de Chagas y sus características ecofisiológicas. Interciencia, 8: 384-95, 1983.

Recebido para publicação em 20.8.1992

Reapresentado em 27.1.1993

Aprovado para publicaçâo em 2.2.1993 